\title{
Propriedades Psicométricas do Mayer-Salovey-Caruso Emotional Intelligence Test - MSCEIT V2.0: Uma Revisão Sistemática da Literatura
}

\author{
Psychometric Properties of the Mayer-Salovey-Caruso Emotional Intelligence Test - \\ MSCEIT V2.0: A Systematic Review of the Literature
}

\author{
Fátima M. Teixeira ${ }^{1}$ e Alexandra M. Araújo ${ }^{2}$
}

\begin{abstract}
Resumo
O presente trabalho pretendeu identificar e examinar criticamente estudos das propriedades psicométricas do Mayer-Salovey-Caruso Emotional Intelligence Test (MSCEIT V2.0) publicados entre 2005 e 2016, em amostras do Brasil, Espanha e Portugal. Para tal, realizou-se uma revisão sistemática da literatura de 14 artigos que testam a validade e a fiabilidade do instrumento, relacionando-o com variáveis como inteligência, personalidade, bem-estar, comportamentos pró-sociais e desempenho profissional. Os estudos com o MSCEIT V2.0 recorreram sobretudo a análises fatoriais exploratórias, assim como a análises da consistência interna através do cálculo do alfa de Cronbach, demonstrando indicadores globais adequados. O instrumento revelou ainda um satisfatório poder preditivo. Sugere-se a revisão do estudo das propriedades psicométricas do MSCEIT V2.0 em outras culturas, de modo a atestar a adequação da medida independentemente do contexto.
\end{abstract}

Palavras-chave: MSCEIT V2.0, inteligência emocional, validade, fiabilidade, revisão sistemática

\begin{abstract}
The present paper aims to identify and critically review studies of the psychometric properties of the MayerSalovey-Caruso Emotional Intelligence Test (MSCEIT V2.0), published between 2005 and 2016, with samples from Brazil, Spain, and Portugal. The systematic review analyzed 14 articles that tested the instrument's validity and reliability, as well as relations with intelligence, personality, well-being, prosocial behavior, and job performance. Studies with the MSCEIT V2.0 mostly used exploratory factor analysis, as well as internal consistency analysis with Cronbach alfas, generally showing adequate coefficients. The instrument also presented satisfactory predictive value. We suggest further reviews of the psychometric properties of the MSCEIT V2.0 in other cultures, in order to demonstrate the adequacy of the measure regardless of the context.
\end{abstract}

Keywords: MSCEIT V2.0, emotional intelligence, validity, reliability, systematic review

\footnotetext{
${ }^{1}$ Mestre em Psicologia Clínica e da Saúde. Departamento de Psicologia e Educação, Universidade Portucalense, Porto, Portugal.

${ }^{2} \mathrm{PhD}$, Professora Auxiliar. Departamento de Psicologia e Educação, Universidade Portucalense, Rua Dr. António Bernardino de Almeida, 541, 4200-072 Porto, Portugal. Tel.: +351225572000. E-mail: amaraujo@upt.pt 


\section{Introdução}

A inteligência emocional (IE) constitui um tópico de interesse na comunidade científica e social mais alargada. Uma rápida pesquisa na $W e b$ of Science com o tópico "emotional intelligence" dá-nos conta de um aumento significativo de publicações na última década, com 14673 citações neste domínio apenas no ano de 2016. O aumento da investigação acerca da IE deve-se, em larga medida, à controvérsia em torno da sua definição e consequente clarificação da sua natureza, por um lado, e à necessidade de aprofundar as relações da IE com variáveis de funcionamento pessoal, académico e profissional, por outro. Com efeito, a investigação tem apontado para a importância da IE na predição da qualidade das relações sociais, desempenho no trabalho, desempenho cognitivo, trabalho emocional, bemestar e sucesso académico (Austin \& Saklofske, 2014; Follesdal \& Hagtvet, 2013; Lopes, 2016; Lopes, Salovey, \& Straus, 2003; Mayer, Roberts, \& Barsade, 2008; Mayer, Salovey, Caruso, \& Cherkasskiy, 2011; Salessi \& Omar, 2016; Schutte, Malouff, Simunek, McKenley, \& Hollander, 2002). A IE parece desenvolver-se com o nível de escolaridade, assistindo-se a uma competência crescente no reconhecimento e gestão das emoções e na expressão emocional com a idade e o nível escolar (Cala \& Castrillón, 2015; Costa \& Faria, 2015; Mayer et al., 2011). Por outro lado, a investigação tem identificado diferenças de género na IE, verificando-se tendencialmente pontuações superiores nas suas provas de avaliação no sexo feminino (FernandezBerrocal, Cabello, Castillo, \& Extremera, 2012; Joseph \& Newman, 2010; Sánchez-Aragón, Retana-Franco, \& Carrasco-Chávez, 2008). Além disso, os estudos têm apontado para relações da IE com variáveis como os traços de personalidade (Andrei, Siegling, Aloe, Baldaro, \& Petrides, 2016; Lopes et al., 2003), a alexitimia (Miguel et al., 2010) e a desejabilidade social (Andrei et al., 2016).

O conceito de IE foi introduzido pelos autores Salovey e Mayer (1990; Mayer \& Salovey, 1993, 1997), que a definiram como uma capacidade inerente à compreensão (pessoal e interpessoal), gestão e utilização de emoções, e regulação de pensamentos e ações. Atualmente, a IE é concetualizada como uma capacidade cognitiva (Mayer \& Salovey, 1997; Mayer et al., 2011) ou combinando esta capacidade com as dimensões da personalidade, motivação e estados afetivos (BarOn, 2006; Petrides \& Furnham, 2003). Todavia, o modelo de IE como capacidade cognitiva tem sido afirmado como muito promissor nesta área de estudos (Antonakis \& Dietz, 2010). Esta conceção entende a IE como uma capacidade cognitiva que permite compreender as emoções e significados emocionais de modo a auxiliar o raciocínio e pensamento, resolução de problemas (Mayer et al., 2011), desenvolvimento de competências sociais e comportamentos adaptativos (Mayer \& Salovey, 1997). Ao contrário do que é defendido pelos modelos mistos, no modelo cognitivo, a IE é independente de traços de personalidade e constitui uma inteligência com identidade própria, que promove a adaptação ao meio e a qualidade do pensamento (Fernández-Berrocal \& Extremera, 2005, 2006; Mayer et al., 2011). Os principais precursores deste ponto de vista são Mayer, Salovey e Caruso (2004, 2008; Mayer \& Salovey, 1997), que desenvolveram uma teoria baseada em quatro fatores integrados hierarquicamente por nível de complexidade, sendo que o primeiro diz respeito à perceção, avaliação e expressão de emoções, o segundo à facilitação de emoções, o terceiro à compreensão e análise de emoções e o quarto à gestão de emoções (Mayer \& Salovey, 1997; Mayer et al., 2004). Além disso, este modelo organiza-se em duas componentes: a experiencial e a estratégica. Da primeira componente fazem parte a perceção emocional (i.e., capacidade do individuo identificar e reconhecer as emoções em si e nos outros) e a facilitação das emoções (i.e., distinguir entre emoções, gerar sentimentos e criar emoções adequadas ao contexto). Por seu lado, integram a segunda componente a compreensão das emoções (i.e., compreender emoções complexas e integrálas no pensamento) e a gestão das emoções (i.e., dirigir e gerar emoções conforme a conveniência do indivíduo) (Mayer \& Salovey, 1997; Mayer et al., 2004; Mayer et al., 2011).

Com base neste modelo teórico de IE, foram desenvolvidos os instrumentos de avaliação Multifactor Emotional Intelligence Scale - MEIS (Mayer, Salovey, \& Caruso, 1997) e o MayerSalovey-Caruso Emotional Intelligence Test - 
MSCEIT V2.0 (Mayer, Salovey, \& Caruso, 2002; Mayer, Salovey, Caruso, \& Sitarenios, 2003). O MSCEIT V2.0 é, assim, o instrumento mais recente de uma série de medidas que avaliam a IE como capacidade e que procuram representar o modelo de quatro fatores, tendo sido desenvolvido com o objetivo de aprimorar as versões anteriores - o MSCEIT 1.1 (Mayer, Salovey, \& Caruso, 1999) e, antes disso, o MEIS (Mayer et al., 1997).

As análises fatoriais conduzidas com este instrumento indicam que este representa os domínios da IE através de uma estrutura de um, dois e quatro fatores (Mayer et al., 2003). Por se tratar de um instrumento adaptado e validado para várias línguas, o MSCEIT V2.0 tornou-se popular na avaliação da IE como capacidade (Cobêro, Primi, \& Muniz, 2006; Curci, Lanciano, Soleti, Zammuner, \& Salovey, 2013; Extremera, Fernández-Berrocal, \& Salovey, 2006; Iliescu, Ilie, Ispas, \& Ion, 2013; Rode et al., 2008; Rossen, Kranzler, \& Algina, 2008).

O teste é composto por 141 itens divididos por oito secções ou subtestes, que se organizam em duas áreas, a experiencial e a estratégica, sendo ainda possível obter-se uma pontuação global de IE (Mayer et al., 2002). A resposta ao MSCEIT V2.0 implica a resolução de problemas relativos a situações que envolvem emoções, sendo que esta resolução de problemas emocionais indica a competência na adaptação emocional aos contextos. A correção do teste é feita através de um critério de consenso geral ou através de um critério estabelecido por especialistas. Na cotação por consenso, são atribuídas cotações superiores a indivíduos que selecionam respostas que foram anteriormente selecionadas por uma maioria de indivíduos. Neste caso, as respostas são cotadas em função da sua correspondência com a amostra normativa. Os resultados são ponderados em função da proporção de indivíduos que na amostra normativa fornecem a mesma resposta. Assim, por exemplo, um indivíduo receberá um resultado de .70 se $70 \%$ da amostra normativa escolher aquela resposta em particular. Por outro lado, a cotação com base em critérios estabelecidos por especialistas é efetuada com recurso ao que estes especialistas consideram ser a resposta correta, sendo que o indivíduo receberá uma cotação em função do grau de concordância entre a sua resposta e a resposta da amostra de especialistas.
Assim, se um indivíduo atribuir uma resposta que $86 \%$ dos especialistas forneceram, receberá uma pontuação de .86 para a resposta. Apesar de alguns problemas serem levantados relativamente à adequação do método de cotação por consenso (Keele \& Bell, 2009; Roberts, Zeidner, \& Matthews, 2001), Mayer et al. (2002) encontraram correlações entre ambos os métodos de cotação que variaram entre $r=.93$ e .99 para o teste total, componentes, áreas e subtestes.

$O$ teste inclui oito subtestes: Faces e Paisagens (para a capacidade de perceber as emoções), Facilitação e Sensação (para a capacidade de facilitar as emoções), Transição e Mistura (para a capacidade de compreender as emoções), e Gestão e Relacionamento (para a capacidade de gerir as emoções). O MSCEIT V2.0 foi originalmente validado numa amostra de 2112 jovens e adultos com idades a partir dos 18 anos, tendo sido recolhidos dados em formato de papel ou de forma computorizada (Mayer et al., 2003). Quanto às propriedades psicométricas do teste original, no estudo da fiabilidade através do teste-reteste observou-se um coeficiente de .93 para a escala geral, .90 para a área experiencial, .88 para a área estratégica, .91 na Perceção de emoções, .79 na Facilitação do pensamento, .80 na Compreensão Emocional e .83 na Gestão emocional, sendo que os coeficientes alfa de Cronbach nas oito subescalas do teste variaram entre .55 e .88 (Mayer et al., 2003).

Estudos realizados com o MSCEIT V2.0 mostram uma associação positiva entre a IE e outras variáveis como a realização académica e laboral, produtividade, qualidade das relações interpessoais, liderança, comportamentos prósociais e bem-estar, associando-se negativamente com a agressividade, delinquência e uso de substâncias, e atuando ainda como fator protetor face ao stresse e toxicodependência (Brackett \& Salovey, 2006; Fernández-Berrocal \& Extremera, 2005; Gardner \& Qualter, 2011; Mayer et al., 2004; Rey \& Extremera, 2014). Mayer et al. (2008) sustentam a necessidade do aperfeiçoamento dos testes de desempenho da IE, visto que estes medem a capacidade de execução dos indivíduos em tarefas mentais e não a crença ou autorrelato dos mesmos acerca dessa sua capacidade. Por outro lado, outros autores (e.g., Karim \& Weisz, 2010; Shao, Doucet, \& Caruso, 
2014) convergem no reconhecimento da importância do estabelecimento da validade intere intracultural das escalas de IE, uma vez que o significado e a vivência das emoções são culturalmente dependentes, sendo uns aspetos mais universais e outros mais culturalmente específicos.

A revisão integrativa conduzida por Joseph e Newman (2010) demonstrou que as medidas de capacidade com base na realização produziam resultados diferenciados das medidas de autorrelato (quer com base em modelos de capacidade quer com base em modelos mistos) quando aferida a validade incremental na predição do desempenho profissional, controlado o efeito da personalidade e da capacidade cognitiva. Este resultado reforça a importância de esclarecer o potencial específico de cada instrumento (ou neste último caso, tipo de instrumento) no estudo da IE, à luz do modelo de IE que o suporta. Neste sentido, o objetivo do presente estudo é apresentar uma revisão sistemática da literatura acerca das propriedades psicométricas do MSCEIT V2.0 (Mayer et al., 2003) como instrumento de avaliação da IE enquanto constructo cognitivo e recorrendo à realização dos indivíduos em tarefas com possibilidade de acerto ou erro. Tendo em conta a importância dos estudos de validação e precisão, assim como da capacidade de $\mathrm{o}$ instrumento se ajustar a determinada cultura, a presente revisão sistemática da literatura procura organizar e comentar as informações relativas às propriedades psicométricas do teste, tendo como base de análise estudos realizados nas populações brasileira, espanhola e portuguesa. Procura-se, assim, identificar o potencial da prova, bem como lacunas de investigação no estudo das suas propriedades, examinando criticamente os estudos publicados.

\section{Método}

A pesquisa realizou-se no mês de abril de 2016, recorrendo a bases de dados bibliográficas (B-On, Web of Science, Scopus, Sciencedirect, RCAAP, Elsevier e Scielo), para a identificação de estudos publicados entre os anos de 2005 e 2016 no domínio da psicologia, em língua portuguesa, inglesa ou espanhola. As palavraschave utilizadas para a pesquisa foram: MSCEIT, inteligência emocional (emotional intelligence), validade (validity) e fidelidade (reliability). Como critérios de inclusão, estabeleceram-se os seguintes: estudos publicados entre 2005 e 2016; estudos com o instrumento MSCEIT V2.0; estudos com o objetivo de estudar as propriedades psicométricas do teste; e estudos realizados com amostras brasileiras, espanholas ou portuguesas. Foram excluídos estudos cujo objetivo central não era o da análise das propriedades do instrumento (e.g., estudos de diferenças de IE entre grupos, estudos de relação da IE com outras variáveis), mesmo que tenham incluído indicadores de fiabilidade ou validade na descrição dos seus resultados. Os estudos foram organizados segundo o ano, país, grupo amostral e tipo de estudo (e.g., estudos de validade, estudos de fiabilidade).

\section{Resultados}

A pesquisa permitiu a identificação de 14 estudos que tinham como objetivo investigar as propriedades psicométricas do instrumento MSCEIT V2.0, com foco quer na validade, quer na fiabilidade (Quadro 1). Assim, identificaram-se dez estudos conduzidos no Brasil, três estudos em Espanha e um em Portugal, sendo que o mais recente foi conduzido com amostra espanhola, tendo sido publicado em 2016.

No que diz respeito aos participantes, em seis dos estudos a amostra foi constituída por estudantes universitários (Bueno et al., 2006; Dantas \& Noronha, 2005, 2006; Jesus Junior \& Noronha, 2007, 2008; Noronha et al., 2007), sendo que um incluiu apenas estudantes universitários de cursos de psicologia (Freitas \& Noronha, 2006) e outro incluiu estudantes universitários e trabalhadores de empresas (Noronha et al., 2007). Um estudo foi realizado junto de alunos do ensino secundário (Marquez et al., 2006) e dois estudos foram conduzidos junto de estudantes do ensino secundário e universitário (Extremera et al., 2006; Sanchez-Garcia et al., 2016). Foram estudadas amostras com funcionários de empresas em dois estudos (Cobêro et al., 2006; Primi et al., 2006). Um estudo foi realizado com militares (Muniz \& Primi, 2007) e um outro estudo utilizou na sua amostra estudantes de uma academia militar (Monteiro, 2009). A média das idades dos participantes 
Quadro 1. Revisão das Propriedades Psicométricas do MSCEIT V2.0

\begin{tabular}{|c|c|c|c|c|}
\hline Autores & País & Grupo amostral & Validade & Fidelidade \\
\hline $\begin{array}{l}\text { Bueno, } \\
\text { Santana, } \\
\text { Zerbini, e }\end{array}$ & Brasil & $\begin{array}{l}\text { Estudantes universitários } \\
(\mathrm{N}=334) \\
M \text { idade }=20.5, D P=3.28\end{array}$ & $\begin{array}{l}\text { Validade de critério (sexo e tipo de curso } \\
\text { superior) }\end{array}$ & $\begin{array}{l}\text { Escala total: } \alpha=.92 ; \text { quatro capacidades: } \\
\alpha>.70 ; \text { componentes experiencial } \mathrm{e} \\
\text { estratégica } \approx .90\end{array}$ \\
\hline
\end{tabular}

(2006)

Cobêro et Brasil Trabalhadores de empresas al., 2006 $(\mathrm{N}=119)$

$M$ idade $=30$ anos $(D P$ sem informação disponível)

\begin{tabular}{|c|c|c|}
\hline $\begin{array}{l}\text { Dantas e } \\
\text { Noronha } \\
(2005)\end{array}$ & Brasil & $\begin{array}{l}\text { Estudantes universitários } \\
(\mathrm{N}=270) \\
M \text { idade }=24 \text { anos, } D P=7.0\end{array}$ \\
\hline $\begin{array}{l}\text { Dantas e } \\
\text { Noronha } \\
(2006)\end{array}$ & Brasil & $\begin{array}{l}\text { Estudantes universitários } \\
(\mathrm{N}=270) \\
M \text { idades }=24 \text { anos, } D P=7.0\end{array}$ \\
\hline $\begin{array}{l}\text { Extremera } \\
\text { et al., } \\
(2006)\end{array}$ & Espanha & $\begin{array}{l}\text { Estudantes do ensino } \\
\text { secundário e universitário } \\
(\mathrm{N}=946) \\
M \text { idade }=19.78 \text { anos, } D P=5.6\end{array}$ \\
\hline
\end{tabular}

Freitas e

Brasil

(2006)

Jesus

Junior e

Noronha

(2007)

Jesus

Junior e

Noronha

(2008)

Márquez,

Martin, e

Brackett

(2006)

$\begin{array}{lll}\text { Monteiro* } & \text { Portugal } & \begin{array}{l}\text { Estudantes da academia } \\ \text { militar } \\ (2009)\end{array} \\ & (\mathrm{N}=106) \\ & M \text { idades }=20 \text { anos, } D P=2.0\end{array}$

$(\mathrm{N}=191)$

$M$ idades $=25$ anos, $D P=7.9$

Brasil Estudantes universitários $(\mathrm{N}=191)$

$M$ idades $=25$ anos, $D P=7.9$ secundário $(\mathrm{N}=77)$ $M$ idades=15.03 anos, $D P=.70$

\section{Validade convergente (inteligência: BPR-5, Não apresenta}

Primi \& Almeida, 1998),

validade discriminante (personalidade: 16

PF, Cattell, Cattell, \& Cattell, 1993) e

validade de critério (desempenho profissional avaliado pelo supervisor e por colegas)

Validade de constructo (análise fatorial exploratória: saturações dos subtestes acima de .64 na componente experiencial e acima de .66 na componente estratégica)

Validade discriminante (personalidade: 16 PF, Rusell \& Karol, 1999)

Validade de critério (sexo e idade)

Consistência interna:

$\alpha$ total=.93;

$.72 \leq \alpha$ capacidades $\leq .89 ; .55 \leq \alpha$ subtestes $\leq .89 ; .78 \leq \alpha$ total por curso (psicologia, biologia, matemática e educação física) $\leq .87$ Não apresenta

Consistência interna: $.69 \leq \alpha \quad$ subtestes consenso $\leq .90 ; \quad .63 \leq \alpha$ subtestes especialista $\leq .89$.

Bipartição de itens:

$r$ total=.95 para cotação consenso; $r$ total=.94 para cotação especialistas; $r$ componente experiencial consenso $=.94 ; r$ componente experiencial especialistas $=.93 ; r$ componente estratégica consenso $=.89 ; r$ componente estratégica especialistas $=.87$; $.82 \leq r$ capacidades consenso $\leq .93 ; .80 \leq r$ capacidades especialistas $\leq .92$.

Consistência interna:

$\alpha$ total $=.88 ; \alpha$ componente experiencial $=.89$; $\alpha$ componente estratégica $=.77 ; .53 \leq \alpha$ capacidades $\leq .87 ; .39 \leq \alpha$ subtestes $\leq .85$. Não apresenta

Validade convergente (inteligência, BPR-5, Primi \& Almeida, 2000)

Validade de constructo (análise fatorial com extração de componentes principais): um fator de componente estratégica com subtestes com pesos fatoriais acima de .54 componente experiencial representada por outros dois fatores, com subtestes com saturações acima de .76

Validade convergente e discriminante: inteligência (Factorial General Intelligence, IGF-5r; Yuste, 2002) e personalidade (Big

Five Questionnaire, BFQ; Caprara, Barbanelli, \& Borgogni, 1993). Validade de critério: competência social comportamento prossocial e mal-apaptativo (Social-Cognitive Attitudes and Strategies, AECS; Moraleda, González, \& GarcíaGallo, 1998) e notas do final do ano. Validade discriminante (personalidade: Inventário Psicológico da Califórnia, Inventário de Personalidade de Eysenck), Validade convergente (inteligência: IG8, versão adaptada das Matrizes Progressivas Avançadas de Raven, 1994); Provas de Aptidão e Realização Cognitiva; Ribeiro, Almeida, Costa, Gaspar, Paiva, Paz, \& Silva, 1994).

Validade de constructo (análise fatorial
Consistência interna: $\alpha$ total $=.90 ; .62 \leq \alpha$ capacidades $\leq .76 ; .51 \leq \alpha$ subtestes $\leq .88$.

Não apresenta

Consistência interna:

$\alpha$ total $=.83, \alpha$ componente experiencial $=.81$, $\alpha$ componente estratégica $=.65, .54 \leq \alpha$ capacidades $\leq .87, .39 \leq \alpha$ subtestes $\leq .85$ 


\begin{tabular}{|c|c|c|c|c|}
\hline $\begin{array}{l}\text { Muniz e } \\
\text { Primi } \\
(2007)\end{array}$ & Brasil & $\begin{array}{l}\text { Polícias militares }(\mathrm{N}=80) \\
M \text { idades }=30.11 \text { anos, } \\
D P=7.40\end{array}$ & $\begin{array}{l}\text { exploratória): identificação de três fatores } \\
\text { (perceção, gestão e facilitação), não } \\
\text { reproduzindo na totalidade o modelo teórico. } \\
\text { Validade de critério: desempenho } \\
\text { profissional (Escala de Avaliação do } \\
\text { Desempenho dos Policiais: Primi, Lima, } \\
\text { Petrini, Nascimento, \& Cruz, 2005) }\end{array}$ & $.45 \leq \alpha$ subtestes $\leq .82$ \\
\hline $\begin{array}{l}\text { Noronha, } \\
\text { Primi, } \\
\text { Freitas, e } \\
\text { Dantas } \\
(2007)\end{array}$ & Brasil & $\begin{array}{l}522 \text { participantes ( } 334 \\
\text { estudantes universitários e } 188 \\
\text { profissionais); } M \\
\text { idades }=23.78, D P=7.54\end{array}$ & - & $\begin{array}{l}.61 \leq \alpha \text { subtestes componente estratégica } \\
\leq .77 ; .05 \leq \text { correlação item - total subteste } \\
\text { Transições } \leq .28 ; .12 \leq \text { correlação item - total } \\
\text { subteste Mistura } \leq .36 ; .01 \leq \text { correlação item - } \\
\text { total subteste Gestão de emoções } \leq .53 ; .04 \leq \\
\text { correlação item - total subteste Relações } \\
\text { emocionais } \leq .44 ;\end{array}$ \\
\hline $\begin{array}{l}\text { Primi, } \\
\text { Bueno, e } \\
\text { Muniz } \\
(2006)\end{array}$ & Brasil & $\begin{array}{l}\text { Funcionários de empresas } \\
(\mathrm{N}=107) \\
\text { Midades }=24.8 \text { anos, } D P=9.9\end{array}$ & $\begin{array}{l}\text { Validade convergente: inteligência (BPR-5; } \\
\text { Primi \& Almeida, 1998, 2000) } \\
\text { Validade discriminante: personalidade } \\
\text { (16PF; Cattell et al., 1993). } \\
\text { Validade de constructo (análise de } \\
\text { componentes principais, com identificação } \\
\text { de dois fatores de inteligência emocional, } \\
\text { bem como fatores quatro fatores que } \\
\text { combinam subtestes da prova com fatores de } \\
\text { inteligência e de personalidade). }\end{array}$ & Não apresenta \\
\hline \multirow[t]{2}{*}{$\begin{array}{l}\text { Sanchez- } \\
\text { Garcia, } \\
\text { Extremera, } \\
\text { e } \\
\text { Fernandez- } \\
\text { Berrocal } \\
(2016)\end{array}$} & Espanha & $\begin{array}{l}\text { Estudo 1: } \\
\text { Estudantes do ensino } \\
\text { secundário e universitário } \\
(\mathrm{N}=3448), \\
M \text { idades }=24.48 \text { anos, } \\
D P=10.67\end{array}$ & $\begin{array}{l}\text { Validade de constructo: análise fatorial } \\
\text { confirmatória, para modelo de oito subtestes, } \\
\text { quatro capacidades, duas componentes e um } \\
\text { resultado global. } \\
\text { Invariância fatorial por sexo. } \\
\text { Validade de critério (sexo). } \\
\text { Validade convergente (método de correção) }\end{array}$ & $\begin{array}{l}\text { Consistência interna: } \\
\alpha \text { total }=.94, \alpha \text { componente experiencial }=.92 \text {, } \\
\alpha \text { componente estratégica }=.98, .71 \leq \alpha \\
\text { subtestes } \leq .92 \text {. } \\
\text { Teste-reteste: } r \geq .70 \text {. }\end{array}$ \\
\hline & & $\begin{array}{l}\text { Estudo } 3 \\
\text { Estudantes universitários } \\
(\mathrm{N}=502), \\
M \text { idades }=21.31 \text { anos, } \\
D P=4.94\end{array}$ & $\begin{array}{l}\text { Validade de critério: } \\
\text { bem-estar (Perceived Well-Being Test; Ryff, } \\
\text { 1989). }\end{array}$ & Não apresenta \\
\hline
\end{tabular}

variou entre os $15.30(D P=.70)$ e 30.11 anos $(D P=7.40)$ e o número de participantes variou entre 80 e 3448 indivíduos.

Quanto à análise da validade do MSCEIT V2.0, os estudos recorreram à análise da validade de constructo, através de análises fatoriais, e análise de validade de critério, validade convergente e validade discriminante. $\mathrm{O}$ estudo da validade fatorial foi efetuado maioritariamente recorrendo a análises fatoriais exploratórias e de retenção de componentes principais (cinco estudos: Dantas \& Noronha, 2005; Jesus Junior \& Noronha, 2008; Monteiro, 2009; Primi et al., 2006; Sanchez-Garcia et al., 2016). Nestes estudos, observou-se que a IE medida pelo MSCEIT V2.0 se diferencia efetivamente de testes de inteligência, de personalidade e de IE percebida (Primi et al., 2006; Sanchez-Garcia et al., 2016), retendo-se as duas áreas experiencial e estratégica da IE (Dantas \& Noronha, 2005). Existe, no entanto, alguma dificuldade em identificar claramente as quatro capacidades avaliadas (Jesus Junior \& Noronha, 2008; Monteiro, 2009), uma vez que as análises tendem a identificar três fatores. Apenas um estudo recorreu à análise fatorial confirmatória, tendo sido ainda avaliada a invariância fatorial em função do sexo (Sanchez-Garcia et al., 2016). Neste caso, foram obtidos indicadores favoráveis de ajuste dos dados (com uma grande amostra: $\mathrm{N}=3448$ ) ao modelo teórico de oito subtestes, quatro capacidades, duas áreas e um resultado 
global, reportando-se ainda que estudantes do sexo masculino e feminino representam o constructo de IE de forma similar.

$O$ estudo da validade convergente do MSCEIT V2.0 recorreu ao uso de testes de inteligência (Côbero et al., 2006; Jesus Junior \& Noronha, 2007; Márquez et al., 2006; Monteiro, 2009; Primi et al., 2006). Foram encontradas correlações moderadas da IE com a inteligência verbal, mas fracas com a inteligência geral, no estudo de Márquez et al. (2006). Por outro lado, observaram-se correlações positivas fracas a moderadas com a inteligência avaliada pela versão da BPR-5 para o Brasil, notando-se que estas correlações se verificam especialmente para o raciocínio abstrato, verbal e espacial (Côbero et al., 2005; Jesus Junior \& Noronha, 2007). Foram ainda encontradas algumas correlações positivas mas fracas (inferiores a .30) entre a IE (ao nível do resultado global, áreas, capacidades e subtestes) e a compreensão verbal, o raciocínio numérico e o raciocínio verbal, numa amostra portuguesa, verificando-se ainda apenas duas correlações com uma medida de inteligência geral (neste caso, para a capacidade compreensão das emoções e para o subteste Mistura) (Monteiro, 2009). Uma análise das correlações encontradas revela que não existe um padrão estável de correlações entre as dimensões avaliadas pelo MSCEIT V2.0 e os diversos tipos de raciocínio avaliados, o que poderá dever-se à qualidade das amostras utilizadas nos estudos. Mais ainda, as correlações não se verificam para todos os subtestes e para todas as capacidades avaliadas.

A validade discriminante utilizou medidas de personalidade (Côbero et al., 2006; Dantas \& Noronha, 2006; Márquez et al., 2006; Monteiro, 2009; Primi et al., 2006; Sanchez-Garcia et al., 2016), de inteligência verbal e geral (Márquez et al., 2006) e uma medida de IE de autorrelato (Sanchez-Garcia et al., 2016). O estudo da validade discriminante com a personalidade mostrou que a IE, tal como medida pelo MSCEIT V2.0, constitui um conceito distinto e que não se sobrepõe à personalidade, tal como demonstrado pelas correlações nulas ou fracas entre os subtestes do MSCEIT V2.0 com os fatores do teste 16 PF (Côbero et al., 2006; Dantas e Noronha, 2006) e com os fatores do California Psychological Inventory e do Eysenk Personality
Inventory (Monteiro, 2009), ou ainda pelas correlações nulas com o neuroticismo, a extroversão e a conscienciosidade, estas avaliadas no estudo de Márquez et al. (2006). Esta validade discriminante foi igualmente demonstrada no estudo de Márquez et al. (2006), onde se verificou que o MSCEIT V2.0 não se correlacionou com a inteligência geral e apenas moderadamente com a inteligência verbal. Através de análise fatorial, foi ainda possível evidenciar que as capacidades avaliadas pelo MSCEIT V2.0 se diferenciam de fatores de IE autorrelatada e da personalidade avaliada em termos dos cinco grandes fatores, extroversão, amabilidade, conscienciosidade, neuroticismo e abertura à experiência (SanchezGarcia et al., 2016). Tal diferenciação foi ainda observada, apesar de com menos clareza, no estudo de Primi et al. (2006), onde se verificou que os oito subtestes do MSCEIT V2.0 se diferenciam, na sua maioria, dos fatores onde estão incluídas as aptidões avaliadas pela BPR-5 e os traços de personalidade avaliados pelo 16PF.

De modo a testar a validade de critério, foram utilizadas as variáveis desempenho profissional (Cobêro et al., 2006; Freitas \& Noronha, 2006; Muniz \& Primi, 2007), competências sociais (Marquez et al., 2006) e bem-estar (SanchezGarcia et al., 2016). Além disso, a validade entre grupos procurou estudar as diferenças entre sexos (Bueno et al., 2006; Extremera et al., 2006; Sanchez-Garcia et al., 2016), grupo etário (Extremera et al., 2006) e curso universitário (Bueno et al., 2006). A validade preditiva foi avaliada através do estudo das relações entre a IE e o rendimento académico no final do ano letivo (Márquez et al., 2006). A IE avaliada com o MSCEIT V2.0 está positivamente associada a níveis mais elevados de comportamento pro-social e negativamente associada a comportamento maladaptativo (Marquez et al., 2006), positivamente associada a níveis mais elevados de desempenho profissional avaliado por supervisor e por colega (Côbero et al., 2006; Freitas \& Noronha, 2006; Muniz \& Primi, 2007), e a uma maior perceção de bem-estar (Sanchez-Garcia et al., 2016). A IE é, ainda, diferente no sexo feminino e masculino, obtendo-se resultados mais elevados no grupo feminino (Bueno et al., 2006; Extremera et al., 2006; Sanchez-Garcia et al., 2016). A IE aumenta com a idade, observando-se resultados mais 
elevados em indivíduos mais velhos (Extremera et al., 2006). Por outro lado, é mais elevada em estudantes cuja formação implica uma maior necessidade de lidar com emoções, como é o caso dos estudantes de psicologia, de comunicação e de artes (Bueno et al., 2006), ou ainda de educação física (Dantas \& Noronha, 2005). Finalmente, os resultados globais de IE avaliados pelo MSCEIT V2.0 predizem positivamente médias de resultados académicos, no final do ano letivo, em estudantes do ensino secundário (Márquez et al., 2006).

A validade incremental do MSCEIT V2.0 foi demonstrada no estudo de Márquez et al. (2006), onde se observou que as relações entre a IE e o comportamento prossocial, a autoconfiança e o rendimento académico se mantêm significativas após ter sido controlado o efeito da personalidade e da inteligência geral. Também no estudo de Sánchez-Garcia et al. (2016) observou-se que a IE medida pelo MSCEIT V2.0 apresenta relações positivas e significativas, tanto em homens como em mulheres, com o bem-estar percebido, depois de controlada a associação deste com os cinco grandes fatores de personalidade.

Relativamente aos estudos que analisaram a fiabilidade do teste, os autores recorreram à análise da consistência interna através do alfa de Cronbach (nove estudos: Bueno et al., 2006; Dantas \& Noronha, 2005; Extremera et al., 2006; Freitas \& Noronha, 2006; Jesus Junior \& Noronha, 2008; Monteiro, 2008; Muniz \& Primi, 2007; Noronha et al., 2007; Sanchez-Garcia et al., 2016). Foram realizados estudos em Espanha que incluíram os métodos de bipartição de itens (Extremera et al. 2006) e de teste-reteste (Sanchez-Garcia et al., 2016). Os estudos que realizaram testes de precisão ao MSCEIT V2.0 revelaram que este se mostra globalmente consistente e homogéneo, com alfas de Cronbach da escala total a variarem entre .78 e .94 , sendo que o teste de bipartição de itens apresenta resultados no mesmo sentido (Extremera et al., 2006). No que respeita à consistência interna dos subtestes, a leitura do Quadro 1 mostra que se observa variabilidade nos resultados, sendo que existem algumas fragilidades nalguns subtestes (com alfas mínimos próximos de .50) e consistência interna muito satisfatória noutros (com alfas máximos a atingirem .89). Os indicadores de consistência interna das quatro capacidades revelam igualmente esta variabilidade: enquanto que parece existir alguma fragilidade nalgumas capacidades, obtendo-se valores mínimos entre .53 e .72 , noutras a consistência interna é muito elevada, chegando a valores próximos de .90 . Os indicadores de consistência interna para as duas componentes são globalmente mais satisfatórios, atingindo na maior parte dos casos valores próximos de .90 . Finalmente, os resultados do teste à estabilidade do MSCEIT V2.0 reforçam a sua precisão, obtendo-se correlações acima de .70 (SanchezGarcia et al., 2016).

Considerando as opções para a cotação das respostas, a cotação escolhida pelos autores dos estudos baseou-se no consenso, ou seja, nas respostas dadas com mais frequência pelos sujeitos da amostra normativa, excetuando o estudo de Extremera et al. (2006), que comparou os resultados por método de consenso e por consulta de especialistas, sendo que o primeiro indica maior fiabilidade como sistema de cotação. Neste aspeto, destaca-se ainda o estudo de Sanchez-Garcia et al. (2016), que avaliou a convergência dos resultados, correlacionando os que foram obtidos por consenso na amostra normativa espanhola com os que se basearam em especialistas $(r=.98)$ e os que se apuraram a partir do consenso dos estudos americanos ( $r=.97$; Mayer et al., 2002).

\section{Discussão}

A presente revisão sistemática da literatura procurou identificar e analisar criticamente os estudos das propriedades psicométricas do MSCEIT V2.0 (Mayer et al., 2003) realizados em território brasileiro, espanhol e português e publicados na última década. As investigações mais recentes sugerem que o modelo de capacidades de IE é o que mais se aproxima de um modelo padrão de inteligência, uma vez que é defendida a combinação de inteligência e emoção, em que esta última está presente na facilitação de processos cognitivos (Mayer et al., 2011). O teste MSCEIT V2.0 é a última versão de um teste correspondente ao modelo teórico desenvolvido pelos pioneiros da IE Mayer et al. (2002), que a descreveram como capacidade cognitiva. O estudo 
das suas propriedades psicométricas em diferentes culturas torna-se importante no sentido de perceber se o teste é capaz de medir efetivamente a IE e de captar diferenças individuais (Mayer et al., 2008), independentemente do contexto cultural onde é administrado.

No presente estudo, verificou-se que o Brasil é o país que apresenta mais estudos publicados no domínio, seguido de Espanha que apresenta dois estudos robustos, sendo que um deles contribuiu para a única e atual versão do instrumento no país. $\mathrm{O}$ único estudo considerado com amostras portuguesas foi publicado numa dissertação de mestrado, ao contrário do ocorrido no Brasil e em Espanha, onde os estudos foram publicados em revistas com revisão por pares. Tanto em Portugal como no Brasil, apesar dos estudos desenvolvidos, não existe uma versão oficial do teste, validada para as respetivas populações. Relativamente às datas das publicações, apesar da última datar de 2016, 13 dos 14 estudos identificados foram conduzidos até 2009, o que parece indicar que atualmente os estudos nos países selecionados para esta revisão centrar-se-ão mais na modelação de relações da IE com outras variáveis do que no estudo das propriedades psicométricas do teste. Dado que se verificaram fragilidades na validade e fiabilidade do teste, esta interrupção no estudo das propriedades do teste condiciona a afirmação do instrumento enquanto medida de IE.

Um fator importante a considerar na análise dos estudos produzidos neste domínio prende-se com a qualidade das amostras, havendo a necessidade de amostras amplas e representativas, de modo a permitir a generalização dos resultados (Almeida \& Freire, 2007). Tendo em conta que o MSCEIT V2.0 se trata de um teste com 141 itens, os estudos aqui contemplados, à exceção do estudo realizado por Sanchez-Garcia et al. (2016), não respondem a essa exigência e poderão colocar em causa as conclusões tiradas com a utilização deste instrumento. Por outro lado, os estudos têm integrado essencialmente estudantes universitários, não contemplando outros setores populacionais, como por exemplo os jovens pouco escolarizados ou os indivíduos seniores. $\mathrm{O}$ enfoque dos estudos na população universitária poderá contribuir para um enviesamento dos resultados encontrados na análise das propriedades do instrumento.
Tendo por base as 14 publicações identificadas para a presente revisão, é possível tecer várias reflexões sobre os estudos das propriedades psicométricas do MSCEIT V2.0. A primeira delas prende-se com a confirmação de que, nas amostras consideradas, a IE medida pelo MSCEIT V2.0, conceptualizada como sendo uma capacidade, não se sobrepõe com constructos como a inteligência (fluida e cristalizada) e a personalidade. Mayer et al. (2004) defendem esta diferenciação, referindo que a IE deve-se correlacionar apenas modestamente com outras medidas de inteligência. Da mesma forma, os traços de personalidade devem ser independentes da IE (Fernández-Berrocal \& Extremera, 2005), não se correlacionando significativamente com nenhum destes, ao contrário do que acontece nas medidas de autorrelato (Mayer et al., 2011). Estes resultados foram globalmente encontrados na presente revisão da literatura, uma vez que se confirmaram correlações nulas ou fracas entre o MSCEIT V2.0 e provas de personalidade e correlações fracas a moderadas com provas de avaliação da inteligência. Efetivamente, os resultados apresentados relativos à validade do MSCEIT V2.0 levam a refletir sobre a explicação teórica da IE como capacidade. Visto que as correlações entre IE, personalidade e inteligência são baixas, a probabilidade de ambas serem variáveis latentes da IE é muito reduzida. Com efeito, os investigadores nesta área alertam para a diferença entre estas duas abordagens: os modelos mistos que definem a IE como traço de personalidade (Petrides \& Furnham, 2003) e os modelos de capacidade, em que a IE representa uma capacidade cognitiva relacionada com as emoções (Mayer et al., 2008). É importante, ainda, notar que os estudos apontam para baixas correlações entre medidas de capacidade e medidas de traço e de autorrelato (Joseph \& Newman, 2010; Mayer et al., 2004; Mayer et al., 2011), o que também foi verificado nesta revisão (Sanchez-Garcia et al., 2016).

Relativamente ao poder preditivo da IE medida com o MSCEIT V2.0, a presente revisão sistemática da literatura mostrou que os resultados obtidos na prova estão positivamente relacionados com o desempenho profissional, a competência social, o bem-estar e o rendimento académico. Estes estudos corroboram estudos prévios que 
demonstraram estas relações com o desempenho profissional (Extremera et al., 2006; Lopes, 2016), com a qualidade das competências sociais, importantes para as interações sociais e adaptação social (Austin \& Saklofske, 2014; Extremera et al., 2006; Mayer et al., 2008), e variáveis da saúde, como o bem-estar subjetivo (Mayer et al., 2011). Este resultado apoia a sua utilização em estudos aplicados aos domínios do trabalho, escolar e da saúde. Por outro lado, em amostras brasileiras e espanholas o sexo feminino mostrouse mais competente na IE, assim como os indivíduos mais velhos, sugerindo que a IE se desenvolve com a idade e é influenciada pelo género, suportando assim os resultados de estudos prévios (Fernández-Berrocal et al., 2012; Mayer et al., 2004; Mayer et al., 2011). Estas conclusões podem apoiar a descrição do que será o perfil de um individuo com maior e menor nível de IE, visto que a IE como capacidade, entre o que já foi referido anteriormente, prediz várias características como a liderança, comportamentos pró-sociais (Mayer et al., 2004, 2008) e maior sensibilidade às emoções em determinadas profissões, como aquelas ligadas à saúde e às artes (Bueno et al., 2006).

Uma apreciação geral do estudo da validade fatorial do instrumento permite-nos observar que apenas um estudo recorreu à análise fatorial confirmatória e ao estudo da invariância fatorial por sexo (Sanchez-Garcia et al., 2016), não se podendo assim garantir a justiça nas comparações efetuadas de resultados de IE de mulheres e de homens nas amostras identificadas. Dadas as vantagens do estudo confirmatório dos modelos de medida na testagem de modelos teóricos, mais estudos deveriam ter procurado incluir a análise fatorial confirmatória no sentido de testar a teoria subjacente ao MSCEIT V2.0 (Rossen et al., 2008). Aliás, os estudos reportados na presente revisão sistemática da literatura permitiram observar algumas fragilidades na demonstração deste modelo de medida, através das análises fatoriais e de componentes principais conduzidas, verificando-se dificuldades em identificar claramente as quatro capacidades de IE. Contudo, o estudo que recorreu à maior amostra e que incluiu análise fatorial confirmatória nos seus procedimentos confirmou a estrutura original do teste de um, dois e quatro fatores, reforçando as diferenças existentes quando adotados diferentes procedimentos de análise da estrutura fatorial do instrumento.

As fragilidades encontradas no estudo da validade de constructo do MSCEIT V2.0 prolongam-se também no estudo da sua fiabilidade. Enquanto o teste global e as duas componentes obtêm indicadores elevados de consistência interna, o mesmo não acontece com tanta clareza no que respeita às quatro capacidades e a todos os oito subtestes. Ainda que os valores máximos de alfas reportados confirmem uma muito satisfatória fiabilidade para alguns subtestes, outros parecem não apresentar suficiente homogeneidade nos itens que os integram. Estudos futuros devem aprofundar o contributo de cada item para o total da subescala, no sentido de identificar os melhores itens na avaliação da IE pelo MSCEIT V2.0. Recorde-se que entre os 14 artigos selecionados para a revisão sistemática da literatura, apenas o estudo de Noronha et al. (2007) estudou as correlações do item com o total corrigido nos subtestes que integram a componente estratégica, tendo identificado itens com correlações muito fracas com o seu subteste. Finalmente, importa referir que os estudos de fidelidade relativos à estabilidade do teste apresentam valores condizentes com o teste original, apontando o MSCEIT V2.0 como um instrumento estável e neste sentido com boa precisão (Mayer et al., 2012).

Concluindo, os estudos identificados na presente revisão da literatura defendem a estrutura original do MSCEIT V2.0 e os seus objetivos de medida na população brasileira, espanhola e portuguesa, apresentando indicadores que suportam, na maioria, a sua validade de constructo, de critério, convergente e discriminante, tal como encontrado noutras culturas (e.g., Rossen et al., 2008). O MSCEIT V2.0 apresenta-se como uma prova com qualidades psicométricas globalmente adequadas, podendo ser utilizada em contexto académico ou organizacional, a partir dos dezassete anos. No entanto, algumas considerações devem ser tidas quanto à fiabilidade do instrumento, antecipandose que existem subtestes onde a homogeneidade não está garantida, necessitando, por isso, de maior estudo no futuro. Quanto às limitações 
desta revisão, estas prendem-se com os critérios de inclusão que apenas incluem estudos com o MSCEIT V2.0 em três países. Futuramente, sugere-se uma revisão que tenha em conta todos os estudos de qualidades psicométricas realizados com o MSCEIT V2.0 nas várias culturas e desde a sua introdução no domínio da avaliação da IE, no sentido de compreender a aplicabilidade do teste e as suas potencialidades de forma mais alargada. Importa, sobretudo, analisar estudos que tenham incluído amostras significativas e adequadas e que tenham incluído nos seus procedimentos as análises estatísticas de que dispomos atualmente, nomeadamente a análise fatorial confirmatória. Por outro lado, importa perceber até que ponto as medidas de capacidade da IE se adequam ou não àquilo que é a $\mathrm{IE}$, contribuindo para $\mathrm{o}$ esclarecimento do conceito e para a forma mais precisa de o mensurar.

\section{Referências}

Almeida, L., \& Freire, T. (2007). Metodologia de investigação em psicologia e educação (4 $4^{\mathrm{a}}$ ed.). Braga: Psiquilibrios.

Andrei, F., Siegling, A. B., Aloe, A. M., Baldaro, B., \& Petrides, K. V. (2016). The incremental validity of the Trait Emotional Intelligence Questionnaire (TEIQue): A systematic review and meta-analysis. Journal of Personality Assessment, 98, 261-276. doi:10.1080/00223891.2015.1084630

Antonakis, J., \& Dietz, J. (2010). Emotional intelligence: On definitions, neuroscience, and marshmallows. Industrial and Organizational Psychology, 3, 165-170. doi:10.1111/j.1754-9434.2010.01219.x

Austin, E. J., \& Saklofske, D. H. (2014). Introduction to the special issue on emotional intelligence. Personality and Individual Differences, 65, 1-2. doi:10.1016/j.paid.2014.02.006

Bar-On, R. (2006). The Bar-On model of emotional-social intelligence (ESI). Psicothema, 18, 13-25.

Benet-Martínez, V., \& John, O. P. (1998). Los Cinco Grandes across cultures and ethnic groups: Multitrait multimethod analyses of the Big Five in Spanish and English. Journal of Personality and Social Psychology, 75, 729-750. http://dx.doi.org/10.1037/0022-3514.75.3.729
Brackett, M. A., \& Salovey, P. (2006). Measuring emotional intelligence with the MayerSalovey-Caruso Emotional Intelligence Test (MSCEIT). Psicothema, 18, 34-41.

Bueno, J. M. H., Santana, P. R. S., Zerbini, J., \& Ramalho, T. B. (2006). Inteligência emocional em estudantes universitários. Psicologia: Teoria e Pesquisa, 22, 305-316. doi:10.1590/S0102-37722006000300007

Cala, M. L. P., \& Castrillón, J. J. C. (2015). Inteligencia emocional y rendimiento académico en estudiantes universitarios. Psicología desde el Caribe, 32, 268-399. doi:10.14482/psdc.32.2.5798

Caprara, G.V., Barbanelli, C., \& Borgogni, L. (1993). Big Five Questionnaire (BFQ). Madrid: TEA.

Cattell, R. B., Cattell, A. K. S., \& Cattell, H. E. P. (1993). Questionário 16PF - Quinta edição. Rio de Janeiro: CEPA.

Cobêro, C., Primi, R., \& Muniz, M. (2006). Inteligência emocional e desempenho no trabalho: Um estudo com MSCEIT, BPR-5 e 16PF. Paidéia, 16, 337-348. doi:10.1590/S0103-863X2006000300005

Costa, A., \& Faria, L. (2015). The impact of emotional intelligence on academic achievement: A longitudinal study in Portuguese secondary school. Learning and Individual Differences, 37, 38-47. doi:10.1016/j.lindif.2014.11.011 1041-6080

Curci, A., Lanciano, T., Soleti, E., Zammuner, V., \& Salovey, P. (2013). Construct validity of the Italian version of the Mayer-SaloveyCaruso Emotional Intelligence Test (MSCEIT) V2.0. Journal of Personality and Assessment, 95, 486-494. doi:10.1080/00223891.2013.778272

Dantas, M. A., \& Noronha, A. P. P. (2005). Inteligência emocional: Parâmetros psicométricos de um instrumento de medida. Estudos e Pesquisas em Psicologia, 5, 59-72.

Dantas, M. A., \& Noronha, A. P. P. (2006). Inteligência emocional: Validade discriminante entre MSCEIT e 16PF. Paidéia, 16, 59-70. doi: 10.1590/S0103-863X2006000100009

Extremera, N., Fernández-Berrocal, P., \& Salovey, P. (2006). Spanish version of the Mayer-Salovey-Caruso Emotional Intelligence Test 
(MSCEIT): Version 2.0: Reliabilities, age and gender differences. Psicothema, 18, 42-48.

Fernández-Berrocal, P., Cabello, R., Castillo, R., \& Extremera, N. (2012). Gender diferences in emotional intelligence: The mediating effect of age. Behavioral Psychology/Psicología Conductual, 20, 77-89.

Fernández-Berrocal, P., \& Extremera, N. (2005). La inteligencia emocional y la educación de las emociones desde el modelo de Mayer y Salovey. Revista Interuniversitaria de Formación del Profesorado, 19, 63-93.

Fernandez-Berrocal, P., \& Extremera, N. (2006). Emotional intelligence: $\mathrm{A}$ theoretical and empirical review of its first 15 years of history. Psicothema, 18, 7-12.

Follesdal, H., \& Hagtvet, K. (2013). Does emotional intelligence as ability predict transformational leadership?. A multilevel approach. The Leadership Quarterly, 24, 747762. doi:10.1016/j.leaqua.2013.07.004

Freitas, F. A., \& Noronha, A. P. P. (2006). Inteligência emocional e avaliação de alunos e supervisores: Evidências de validade. Psicologia: Teoria e Prática, 8(1), 77-93.

Gardner, K. J., \& Qualter, P. (2011). Factor structure, measurement invariance, and structural invariance of the MSCEIT V2.0. Personality and Individual Differences, 51, 492-496. doi:10.1016/j.paid.2011.05.004

Iliescu, D., Ilie, A., Ispas, D., \& Ion, A. (2013). Examining the psychometric properties of the Mayer-Salovey-Caruso Emotional Intelligence Test: Findings from an Eastern European culture. European Journal of Psychological Assessment, 29, 121-128. doi:10.1027/10155759/a000132

Jesus Junior, A. G., \& Noronha, A. P. P (2007). Inteligência emocional e provas de raciocínio: Um estudo correlacional. Psicologia: Reflexão e Critica, 20, 480-489. doi:10.1590/S0102-79722007000300016

Jesus Junior, A. G., \& Noronha, A. P. P. (2008). Parâmetros psicométricos do Mayer Salovey Caruso Emotional Intelligence Test MSCEIT. PSIC: Revista da Vetor Editora, 9, 145-153.

Joseph, D. L., \& Newman, D. A. (2010). Emotional intelligence: An integrative meta- analysis and cascading model. Journal of Applied Psychology, 95, 54-78.

doi:10.1037/a0017286

Karim, J., \& Weisz, R. (2010). Cross-cultural research on the reliability and validity of the Mayer-Salovey-Caruso Emotional Intelligence Test (MSCEIT). Cross-Cultural Research: The Journal of Comparative Social Science, 44, 374-404. doi:10.1177/1069397110377603.

Keele, S. M., \& Bell, R. C. (2009). Consensus scoring, correct responses and reliability of the MSCEIT V2. Personality and Individual Differences, 47, 740-747. doi:10.1016/j.paid.2009.06.013

Lopes, P. N. (2016). Emotional intelligence in organizations: Bridging research and practice. Emotion Review, 8, 316-321. doi:10.1177/1754073916650496

Lopes, P. N., Salovey, P., \& Straus, R. (2003). Emotional intelligence, personality, and the perceived quality of social relationships. Personality and Individual Differences, 35, 641-658. doi:10.1016/S0191-8869(02)00242-8

Márquez, P. G.-O., Martín, R.P., \& Brackett, M. A. (2006). Relating emotional intelligence to social competence and academic achievement in high school students. Psicothema, 18, 118123.

Mayer, J. D., Roberts, R. D., \& Barsade, S. G. (2008). Human abilities: Emotional intelligence. Annual Review of Psychology, 59, 507-536. doi:10.1146/annurev.psych.59.103006.093646

Mayer, J. D., \& Salovey, P. (1993). The intelligence of emotional intelligence. Intelligence, 17, 433-442. doi:10.1016/0160-2896(93)90010-3

Mayer, J. D., \& Salovey, P. (1997). What is emotional intelligence?. In P. Salovey \& D. J. Sluyter (Eds.), Emotional development and emotional intelligence (pp. 3-31). New York: Basic Books.

Mayer, J. D., Salovey, P., \& Caruso, D. R. (1997). Emotional IQ test [CD ROM]. Needham, MA: Virtual Knowledge.

Mayer, J. D., Salovey, P., \& Caruso, D. R. (1999). MSCEIT item booklet [research version 1.1.]. Toronto, Canada: Multi-Health Systems.

Mayer, J. D., Salovey, P., \& Caruso, D. (2002). Mayer-Salovey-Caruso Emotional Intelligence Test 
(MSCEITC V2.0): User's manual. Toronto, Ontario, Canada: MHS Publishers.

Mayer, J. D., Salovey, P., \& Caruso, D. R. (2004). Emotional intelligence: Theory, findings, and implications. Psychological Inquiry, 15, 197215. doi:10.1207/s15327965pli1503_02

Mayer, J. D., Salovey, P., \& Caruso, D. R. (2008). Emotional intelligence: New ability or eclectic traits?. American Psychologist, 63, 503-517. doi:10.1037/0003-066X.63.6.503.

Mayer, J. D., Salovey, P., \& Caruso, D. R. (2012). The validity of the MSCEIT: Additional analyses and evidence. Emotion Review, 4, 403-408. doi:10.1177/1754073912445815

Mayer, J. D., Salovey, P., Caruso, D. R., \& Cherkasskiy, L. (2011). Emotional intelligence. In R. J. Sternberg \& S. B. Kaufman (Eds.), The Cambridge handbook of intelligence (pp. 528-549). New York: Cambridge University Press.

Mayer, J. D., Salovey, P., Caruso, D. R., \& Sitarenios, G. (2003). Measuring emotional intelligence with the MSCEIT V2.0. Emotion, 3, 97-105. doi:10.1037/1528-3542.3.1.97

Miguel, F. K., Bueno, J. M. H., Noronha, A. P. P., Couto, G., Primi, R., \& Muniz, M. (2010). Alexitimia e inteligência emocional: Estudo correlacional. Psicologia: Teoria e Prática, $12,52-65$.

Monteiro, N. (2009). Inteligência emocional: Validação de constructo do MSCEIT numa amostra portuguesa. Dissertação de mestrado não publicada. Lisboa: Universidade de Lisboa.

Moraleda, M., González Galán, A., \& GarcíaGallo, J. (1998). Cuestionario Actitudes y Estrategias Cognitivosociales (AECS). Madrid: TEA Ediciones.

Muniz, M., \& Primi, R. (2007). Inteligência emocional e desempenho em policiais militares: Validade de critério do MSCEIT. Revista Aletheia, 25, 66-81.

Noronha, A. P. P., Primi, R., Freitas, F. A., \& Dantas, M. A. (2007). Análise dos itens do Mayer-Salovey-Caruso Emotional Intelligence Test: Escalas da área estratégica. Revista Psicologia em Estudo, 12, 415-422.

Petrides, K. V., \& Furnham, A. (2003). Trait emotional intelligence: Behavioural validation in two studies of emotion recognition and reactivity to mood induction. European Journal of Personality, 17, 39-57.

doi:10.1002/per.466

Primi, R., \& Almeida, L. S. (1998). Baterias de Provas de Raciocínio-BPR-5. São Paulo: Casa do Psicólogo.

Primi, R., \& Almeida, L. S. (2000). Baterias de provas de raciocínio (BPR-5): Manual Técnico. São Paulo, SP: Casa do Psicólogo.

Primi, R., Bueno, J. M. H., \& Muniz, M. (2006). Inteligência emocional: Validade convergente e discriminante do MSCEIT com a BPR-5 e o 16 PF. Psicologia Ciência e Profissão, 26, 2645.

Primi, R., Lima, J., Petrini, M. C., Nascimento, M. M., \& Cruz, M. B. Z. (2005). Indicadores para avaliação de desempenho de policiais. Encontro: Revista de Psicologia, 10(12), 5870.

Raven, J. (1994). Occupational user's guide: Raven's Advanced Progressive Matrices \& Mill Hill Vocabulary Scale. Oxford, UK: Oxford Psychologists Press Ltd.

Roberts, R. D., Zeidner, M., \& Matthews, G. (2001). Does EI meet traditional standards for an intelligence? Some new data and conclusions. Emotion, 1, 196-231. doi:10.1037//1528-3542.1.3.196

Salessi, S., \& Omar, A. (2016). Desarollo y validación de una escala para medir actuación emocional en el trabajo. Revista Iberoamericana de Diagnóstico y Evaluación - e Avaliação Psicológica, 41(1), 66-79.

Sánchez-Aragón, R., Retana-Franco, B. E., \& Carrasco-Chávez, E. (2008). Evaluación psicológica del entedimiento emocional: Diferencias y similitudes entre hombres $\mathrm{y}$ mujeres. Revista Iberoamericana de Diagnóstico y Evaluación - e Avaliação Psicológica, 26(2), 193-216.

Rey, L., \& Extremera, N. (2014). Positive psychological characteristics and interpersonal forgiveness: Identifying the unique contribution of emotional intelligence abilities, Big Five traits, gratitude and optimism. Personality and Individual Differences, 68, 199-204. doi:10.1016/j.paid.2014.04.030

Ribeiro, I. S., Almeida, L. S., Costa, A. R., Gaspar, A., Paiva, L., Paz, J., \& Silva, I. 
(1994). Provas de Avaliação da Realização Cognitiva (PARC): Características métricas dos itens e dos resultados. In L. S. Almeida \& I. S. Ribeiro (Orgs.), Avaliação psicológica: Formas e contextos II. Braga: APPORT.

Rode, J. C., Mooney, C. H., Arthaud-Day, M. L., Near, J. P., Rubin, R. S., Baldwin, T. T., \& Bommer, W. H. (2008). An examination of the structural, discriminant, nomological, and incremental predictive validity of the MSCEIT V2.0. Intelligence, 36, 350-366. doi:10.1016/j.intell.2007.07.002

Rossen, E., Kranzler, J. H., \& Algina, J. (2008). Confirmatory factor analysis of the MayerSalovey-Caruso Emotional Intelligence Test V 2.0 (MSCEIT). Personality and Individual Differences, 44, 1258-1269. doi:10.1016/j.paid.2007.11.020

Russell, M. T., \& Karol, D.L. (1999). Manual do 16 PF- Quinta edição. Rio de Janeiro: Centro Editor de Psicologia Aplicada - CEPA.

Ryff, C. D. (1989). Happiness is everything, or is it? Explorations on the meaning of psychological well-being. Journal of Personality and Social Psychology, 57, 10691081. doi:10.1037/0022-3514.57.6.1069

Salovey, P., \& Mayer, J. D. (1990). Emotional intelligence. Imagination, Cognition, and Personality, 9, 185-211.

Sanchez-Garcia, M., Extremera, N., \& FernandezBerrocal, P. (2016). The factor structure and psychometric properties of the Spanish version of the Mayer-Salovey-Caruso Emotional Intelligence Test. Psychological Assessment, $\quad 28, \quad$ 1404-1415. doi:10.1037/pas0000269

Schutte, N. S., Malouff, J. M., Simunek, M., McKenley, J., \& Hollander, S. (2002). Characteristic emotional intelligence and emotional well-being. Cognition and Emotion, 16, 769-785. doi:10.1080/02699930143000482

Shao, B., Doucet, L., \& Caruso, D. R. (2014). Universality versus cultural specificity of three emotion domains: Some evidence based on the cascading model of emotional intelligence. Journal of Cross-Cultural Psychology, $\quad$ 46, 229-251. doi: $10.1177 / 002202211455747$

Yuste, C. (2002). Inteligencia general factorial Revisada 5 (IGF-r 5). Madrid: EOS. 